\title{
Errors in the Measurement of Cross-Correlated Relaxation Rates and How to Avoid Them
}

\author{
T. Carlomagno and C. Griesinger ${ }^{1}$ \\ Institut für Organische Chemie, Universität Frankfurt, Marie-Curie Strasse 11, D-60439 Frankfurt, Germany
}

Received October 19, 1999; revised February 17, 2000

Cross-correlated relaxation rates $\Gamma$ are commonly obtained from constant time experiments by measuring the effect of the desired cross-correlated relaxation on an appropriate coherence during the constant time $T$. These measurements are affected by systematic errors, which derive from undesired cross-correlated relaxation effects taking place before and after the constant time period $T$. In this paper we discuss the sources and the size of these errors in an example of two pulse sequences. Higher accuracy of the measured data can be obtained by recording a set of experiments with different $T$ values. Cross-correlated relaxation rates are measured in constant time experiments either from the differential relaxation of multiplet components $(J$-resolved $\Gamma$ experiments) or from the efficiency of magnetization transfer between two coherences (quantitative $\Gamma$ experiments). In this paper we calculate analytically the statistical errors in both $J$-resolved and quantitative $\Gamma$ experiments. These formulae provide the basis for the choice of the most efficient experimental approach and parameters for a given measurement time and size of the rate. The optimal constant time $T$ for each method can be calculated and depends on the relaxation properties of the molecule under investigation. Moreover, we will show how to optimize the relative duration of cross and reference experiments in a quantitative $\Gamma$ approach. $\odot 2000$ Academic Press

Key Words: cross-correlated relaxation; constant time; systematic errors; $J$-resolved $\Gamma$ experiment; quantitative $\Gamma$ experiment.

\section{INTRODUCTION}

Recently, a number of liquid-state NMR experiments have been developed to derive cross-correlated relaxation rates that occur because of the interference between either two dipolar relaxation mechanisms (1-5) or a dipolar and a CSA relaxation mechanism $(6,7)$ in proteins. These cross-correlated relaxation rates have been demonstrated to be a powerful tool for obtaining both motional and structural information.

Cross-correlated relaxation rates $\Gamma$ can be measured in two ways. In the first type of experiments, which we call " $J$ resolved $\Gamma$ experiments," the rate of interest is extracted from the difference in the intensities of multiplet components which

\footnotetext{
${ }^{1}$ To whom correspondence should be addressed. (Alternate address: Max Planck Institute for Biophysical Chemistry, Am Faßberg 11, 37077 Göttingen, Germany.) Fax: +49-69-79829128. E-mail: cigr@org.chemie.uni-frankfurt.de.
}

have evolved in the presence of cross-correlated relaxation for a constant time period $T$. In the second type of experiments, the "quantitative $\Gamma$ experiments," an initial operator $\hat{A}$ is transferred to an operator $\hat{B}$ by the desired cross-correlated relaxation mechanism. The intensity of this transfer, normalized to an alternative transfer from $\hat{A}$ to $\hat{B}$ with known efficiency, gives a measure of the cross-correlated relaxation rate $(8-14)$.

In the first part of the paper, we analyze sources of systematic errors, affecting both $J$-resolved and quantitative $\Gamma$ experiments, and suggest a way to extract the cross-correlated relaxation rate from experimental data taking these errors into account. This is particularly important for rates of small size, for which accuracy is very critical. In the second part of the paper, we calculate analytical expressions for the statistical errors in $J$-resolved and quantitative $\Gamma$ experiments. These calculations provide the tools for choosing between the two methods and optimizing the constant time $T$ depending on the relaxation properties of the molecule under investigation and on the size of the rate to be measured.

\section{CROSS-CORRELATED RELAXATION RATES FROM $J$-RESOLVED $\Gamma$ AND QUANTITATIVE $\Gamma$ EXPERIMENTS}

\section{Theory}

In $J$-resolved $\Gamma$ experiments, the cross-correlated relaxation rate of interest is obtained from the difference in the intensities of multiplet components. If the cross-correlated relaxation rate $\Gamma_{i, j k}^{\mathrm{CSADD}}$ between the CSA of spin $i$ and the dipolar interaction of spins $j$ and $k$ is to be measured, double and zero quantum coherences of spins $i$ and $j$ evolve for a constant time period $T$ in the presence of $\Gamma_{i, j k}^{\mathrm{CSADD}}$ and $J_{i, k}$ or/and $J_{j, k}$. The two families of coherences, $I_{i}^{ \pm} I_{j}^{ \pm} I_{k}^{\alpha}$ and $I_{i}^{ \pm} I_{j}^{ \pm} I_{k}^{\beta}$, which correspond to the two possible polarization states of spin $I_{k}$, have different relaxation properties. At the end of the constant time period $T$ the intensities of the two lines representing these coherences, $\operatorname{In}_{\alpha}$ and $\operatorname{In}_{\beta}$, are

$$
\begin{aligned}
& \operatorname{In}_{\alpha}=\operatorname{In}_{0 \alpha} e^{-\left(\Gamma^{\mathrm{a}}+\Gamma_{i, j k}^{\mathrm{CS} k \mathrm{DD})}\right) T} \\
& \operatorname{In}_{\beta}=\operatorname{In}_{0 \beta} e^{-\left(\Gamma^{\mathrm{a}-} \Gamma_{i, j k}^{\mathrm{CSADD}}\right) T},
\end{aligned}
$$


where $\mathrm{In}_{0 \alpha}$ and $\mathrm{In}_{0 \beta}$ are the intensities of the respective lines at constant time $T=0$ and $\Gamma^{\mathrm{a}}$ is the contribution due to the autocorrelated relaxation rates, which equally affect the two multiplet components. The possibility of extracting the $\Gamma_{i, j k}^{\mathrm{CSA} / \mathrm{DD}}$ rate from one experiment with a single constant time $T$, according to the equation

$$
\Gamma_{i, j k}^{\mathrm{CSA} / \mathrm{DD}}=\frac{1}{2 T} \ln \frac{\mathrm{In}_{\beta}}{\mathrm{In}_{\alpha}},
$$

relies on the condition $\operatorname{In}_{0 \alpha}=\mathrm{In}_{0 \beta}$, which is not necessarily fulfilled. To make the measurement independent of the initial intensities $\operatorname{In}_{0 \alpha}$ and $\mathrm{In}_{0 \beta}$ and to avoid systematic errors, one can record a series of experiments with different $T$ values and extract the cross-correlated relaxation rates according to

$$
\frac{1}{2} \ln \frac{\mathrm{In}_{\beta}}{\operatorname{In}_{\alpha}}=\frac{1}{2} \ln \frac{\mathrm{In}_{0 \beta}}{\operatorname{In}_{0 \alpha}}+\Gamma_{i, j k}^{\mathrm{CSA} / \mathrm{DD}} T
$$

This analysis is particularly useful for cross-correlated relaxation rates of small amplitude, such as the $\Gamma_{\mathrm{C}^{\prime}, \mathrm{NH}}^{\mathrm{CSA}}+\Gamma_{\mathrm{N}, \mathrm{C}^{\prime} \mathrm{H}^{\mathrm{N}}}^{\mathrm{CSA}}(6)$ and the $\Gamma_{\mathrm{C}^{\prime}, \mathrm{C}^{\prime} \mathrm{C}_{\alpha}}^{\mathrm{CA} \mathrm{AD}}(7)$, which are measured in peptide planes in a $\mathrm{HNCO}$ correlation. In the following, we give an estimate of the size of the systematic errors of the rates derived from such HNCO correlations.

Possible Inaccuracy in the Measurement of $\Gamma_{N, N H^{N}}^{C S A D D}+\Gamma_{C^{\prime}, C^{\prime} H^{N}}^{C S A D D}$
and $\Gamma_{C^{\prime}, N H^{N}}^{C S A D D}+\Gamma_{N, C^{\prime} H^{N}}^{C S A D D}$ The $\Gamma_{\mathrm{N}, \mathrm{NH}}^{\mathrm{CSA}}+\Gamma_{\mathrm{C}^{\prime}, \mathrm{C}^{\prime} \mathrm{H}^{\mathrm{N}}}^{\mathrm{CSADD}}$ and $\Gamma_{\mathrm{C}^{\prime} \mathrm{NH}}^{\mathrm{CSADD}}+\Gamma_{\mathrm{N}, \mathrm{C}^{\prime} \mathrm{H}^{\mathrm{N}}}^{\mathrm{CSADD}}$ rates can be measured by the HNCO-type experiment proposed in Ref. (6). A $3 \mathrm{D}$ version of the sequence is shown in Fig. 1. During the constant time $T \mathrm{DQ}$ and $\mathrm{ZQ} \mathrm{NC}^{\prime}$ coherences evolve under the influence of relaxation; scalar coupling ${ }^{2} J_{\mathrm{C}^{\prime} \mathrm{H}^{\mathrm{N}}} \pm{ }^{1} J_{\mathrm{NH}^{\mathrm{N}}}$ and chemical shifts $\Omega_{\mathrm{C}^{\prime}} \pm \Omega_{\mathrm{N}}$ evolve in $t_{1}$, giving rise to two doublets in $\omega_{1}$. Two cross-correlated relaxation rates, $\Gamma^{\mathrm{DQ}}$ and $\Gamma^{\mathrm{ZQ}}$, can be extracted from the intensity difference of the two lines of the DQ and ZQ doublets. The $\Gamma_{\mathrm{N}, \mathrm{NH}^{\mathrm{N}}}^{\mathrm{CS} / \mathrm{DD}}+\Gamma_{\mathrm{C}^{\prime}, \mathrm{C}^{\prime} \mathrm{H}^{\mathrm{N}}}^{\mathrm{CA} / \mathrm{DD}}$ and $\Gamma_{\mathrm{C}^{\prime}, \mathrm{NH}}^{\mathrm{CSADD}}+\Gamma_{\mathrm{N}, \mathrm{C}^{\mathrm{N}} \mathrm{H}^{\mathrm{N}}}^{\mathrm{CS} / \mathrm{DD}}$ are obtained by adding and subtracting $\Gamma^{\mathrm{DQ}}$ and $\Gamma^{\mathrm{ZQ}}$, according to

$$
\begin{aligned}
& \Gamma_{\mathrm{N}, \mathrm{NH} H^{\mathrm{N}}}^{\mathrm{CSA} / \mathrm{DD}}+\Gamma_{\mathrm{C}^{\prime}, \mathrm{C}^{\prime} \mathrm{H}^{\mathrm{N}}}^{\mathrm{CS} / \mathrm{DD}}=\frac{1}{2}\left(\Gamma^{\mathrm{DQ}}+\Gamma^{\mathrm{ZQ}}\right) \\
& \Gamma_{\mathrm{C}^{\prime}, \mathrm{NH}}^{\mathrm{CSA} / \mathrm{DD}}+\Gamma_{\mathrm{N}, \mathrm{C}^{\prime} \mathrm{H}^{\mathrm{N}}}^{\mathrm{CS} / \mathrm{DD}}=\frac{1}{2}\left(\Gamma^{\mathrm{DQ}}-\Gamma^{\mathrm{ZQ}}\right) .
\end{aligned}
$$

The analysis of the evolution of the relevant operators is given in Fig. 1. Depending on the efficiency $s$ of the WALTZ-16 decoupling sequence in mixing the $\mathrm{H}_{\alpha}^{\mathrm{N}}$ and the $\mathrm{H}_{\beta}^{\mathrm{N}}$ states, the following line intensities are measured:

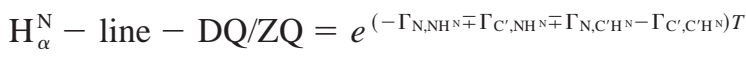

$$
\begin{aligned}
& \times\left[(1-s)^{2} e^{-\Gamma_{\mathrm{N}, \mathrm{NH} N} 2 \Delta}+2 s(1-s)+s^{2} e^{\Gamma_{\mathrm{N}, \mathrm{NH} N 2 \Delta}}\right] \\
& \mathrm{H}_{\beta}^{\mathrm{N}}-\text { line }-\mathrm{DQ} / \mathrm{ZQ}=e^{\left(-\Gamma_{\mathrm{N}, \mathrm{NH}} \pm \Gamma_{C^{\prime}, \mathrm{NH}} \pm \Gamma_{\mathrm{N}^{\prime} \mathrm{C}^{\mathrm{N}} \mathrm{N}}+\Gamma_{\left.\mathrm{C}^{\prime}, \mathrm{C}^{\prime} \mathrm{H}^{\mathrm{N}}\right) T}\right.} \\
& \times\left[(1-s)^{2} e^{\Gamma_{\mathrm{N}, \mathrm{NH} N 2 \Delta}}+2 s(1-s)+s^{2} e^{-\Gamma_{\mathrm{N}, \mathrm{NH} N 2 \Delta}}\right] .
\end{aligned}
$$

The first factor carries the desired cross-correlated relaxation rates and the second factor corresponds to different initial line intensities $\operatorname{In}_{0 \alpha}$ and $\operatorname{In}_{0 \beta}$. Depending on $s$, the term $1 / 2 \ln \operatorname{In}_{0 \beta} / \mathrm{In}_{0 \alpha}$ varies between $\pm 2 \Gamma_{\mathrm{N}, \mathrm{NH}} \Delta= \pm 0.05$ for $\Gamma_{\mathrm{N}, \mathrm{NH}^{\mathrm{N}}}=4.5 \mathrm{~Hz}$.

In our experiment the proton decoupling was turned on for $8.1 \mathrm{~ms}$, which corresponded to about 1.5 cycles of the WALTZ-16 sequence. With these parameters $s$ was close to 1 on resonance, leading to the maximum possible value for $1 / 2 \mathrm{ln}$ $\mathrm{In}_{0 \beta} / \mathrm{In}_{0 \alpha}$. The effect of this is shown in Fig. 2 for the peptide plane Lys29-Thr30 of the protein ubiquitin. In the upper and lower parts of the figure the $\operatorname{In}_{\beta}^{\mathrm{ZQ}} / \mathrm{In}_{\alpha}^{\mathrm{ZQ}}$ and $\operatorname{In}_{\beta}^{\mathrm{DQ}} / \mathrm{In}_{\alpha}^{\mathrm{DQ}}$ intensities ratios are shown as functions of the constant time $T$, respectively. The data are best fitted with a coefficient $1 / 2 \ln \operatorname{In}_{0 \beta} / \mathrm{In}_{0 \alpha}$ of 0.04 for both the DQ and the ZQ doublet. The rates which one obtains are $\Gamma_{\mathrm{N}, \mathrm{NH}}^{\mathrm{CS} / \mathrm{DD}}+\Gamma_{\mathrm{C}^{\prime}, \mathrm{C}^{\prime} \mathrm{H}^{\mathrm{N}}}^{\mathrm{CS} / \mathrm{DD}}=5.7 \pm 0.2 \mathrm{~Hz}$ and $\Gamma_{\mathrm{C}^{\prime}, \mathrm{NH}}^{\mathrm{CSADD}}$ $+\Gamma_{\mathrm{N}, \mathrm{C}^{\mathrm{N}} \mathrm{H}^{\mathrm{N}}}^{\mathrm{CS} / \mathrm{DD}}=-1.6 \pm 0.2 \mathrm{~Hz}$. On the other hand, if one assumes that $1 / 2 \ln \operatorname{In}_{0 \beta} / \mathrm{In}_{0 \alpha}=0$, the cross-correlated relaxation rates are $\Gamma_{\mathrm{N}, \mathrm{NH}}^{\mathrm{CS} / \mathrm{DD}}+\Gamma_{\mathrm{C}^{\prime}, \mathrm{C}^{\prime} \mathrm{H}^{\mathrm{N}}}^{\mathrm{CS} / \mathrm{DD}}=6.2 \pm 0.2 \mathrm{~Hz}$ and $\Gamma_{\mathrm{C}^{\prime}, \mathrm{NH}^{\mathrm{N}}}^{\mathrm{CSA} / \mathrm{DD}}+\Gamma_{\mathrm{N}, \mathrm{C}^{\prime} \mathrm{H}^{\mathrm{N}}}^{\mathrm{CSA} / \mathrm{DD}}=$ $-1.6 \pm 0.2 \mathrm{~Hz}$. For the $\Gamma_{\mathrm{C}^{\prime}, \mathrm{NH}}^{\mathrm{CS} / \mathrm{DD}}+\Gamma_{\mathrm{N}, \mathrm{C}^{\prime} \mathrm{H}^{\mathrm{N}}}^{\mathrm{CS} / \mathrm{DD}}$ rate, which is obtained from the difference of $\Gamma^{\mathrm{DQ}}$ and $\Gamma^{\mathrm{ZQ}}$, the two systematic errors on the $\Gamma^{\mathrm{DQ}}$ and $\Gamma^{\mathrm{ZQ}}$ mutually cancel out, while they add up for the $\Gamma_{\mathrm{N}, \mathrm{NH}}^{\mathrm{CSA} N \mathrm{ND}}+\Gamma_{\mathrm{C}^{\prime} \mathrm{C}^{\prime} \mathrm{H}^{\mathrm{N}}}^{\mathrm{CSA}}$ rate, which is obtained from the sum of $\Gamma^{\mathrm{DQ}}$ and $\Gamma^{\mathrm{ZQ}}$. This results in an error of $0.5 \mathrm{~Hz}$ for $\Gamma_{\mathrm{N}, \mathrm{NH}}^{\mathrm{CSA} / \mathrm{DD}}+$ $\Gamma_{\mathrm{C}^{\prime}, \mathrm{C}^{\prime} \mathrm{H}^{\mathrm{N}}}^{\mathrm{CSAIDD}}$, which is equal to $10 \%$ of the measured value.

The coefficient $1 / 2 \ln \operatorname{In}_{0 \beta} / \operatorname{In}_{0 \alpha}$ would be equal to zero if the effect of the cross-correlated relaxation rate $\Gamma_{\mathrm{N}, \mathrm{NHN}}$ during the two delays $\Delta$ was purged by a $90^{\circ}$ proton pulse applied before the beginning of the constant time $T$. This pulse sequence might allow the measurement of the two $\Gamma_{\mathrm{N}, \mathrm{NH}^{\mathrm{N}}}^{\mathrm{CSADD}}+\Gamma_{\mathrm{C}^{\prime}, \mathrm{C}^{\prime} \mathrm{H}^{\mathrm{N}}}^{\mathrm{CSADD}}$ and $\Gamma_{\mathrm{C}^{\prime}, \mathrm{NH}}^{\mathrm{CS} / \mathrm{DD}}+\Gamma_{\mathrm{N}, \mathrm{C}^{\prime} \mathrm{H}^{\mathrm{N}}}^{\mathrm{CSA} / \mathrm{d}}$ rates from a single experiment.

\section{Possible Inaccuracy in the Measurement of $\Gamma_{C^{\prime}, C^{\prime} C_{\alpha}}^{C A / D}$}

Similarly to what was described in the previous paragraph, the $\Gamma_{\mathrm{C}^{\prime}, \mathrm{C}^{\prime} \mathrm{C}_{\alpha}}^{\mathrm{CSA} D \mathrm{DD}}$ rate, measured in the $\mathrm{C}_{\alpha}$-coupled $\mathrm{HNCO}$ experiment proposed in Ref. (7) (Fig. 3), is affected by systematic errors as well. The cross-correlated relaxation rate $\Gamma_{\mathrm{C}^{\prime}, C^{\prime} C_{\alpha}}^{\mathrm{CSADD}}$ is active for the entire delays $2 \tau\left(J_{\mathrm{NC}^{\prime}}\right.$ defocusing and refocusing delays) before and after the constant time period $T$. During these delays the magnetization of the carbonyl nuclei is longitudinal and the frequency-independent spectral density term $J(0)$ of the cross-correlated relaxation rate has no effect on it. On the other hand, due to the effect of the spectral density term of the $\Gamma_{\mathrm{C}^{\prime}, C^{\prime} C_{\alpha}}^{\mathrm{CSADD}}$ at frequency $\omega_{\mathrm{C}}$, the following operators are present at point $a$ :

$$
\begin{aligned}
& \mathrm{C}_{y}^{\prime} \mathrm{C}_{\alpha}(\alpha) e^{2 \Gamma_{C^{\prime}, C^{\prime} C_{\alpha}}^{\mathrm{CSA} D} \tau} \\
& \mathrm{C}_{y}^{\prime} \mathrm{C}_{\alpha}(\beta) e^{-2 \Gamma_{C^{\prime}, \mathrm{C}^{\prime} C_{\alpha}}^{\mathrm{CS} / / \mathrm{D}} \tau} \text {. }
\end{aligned}
$$


$\mathbf{A}$

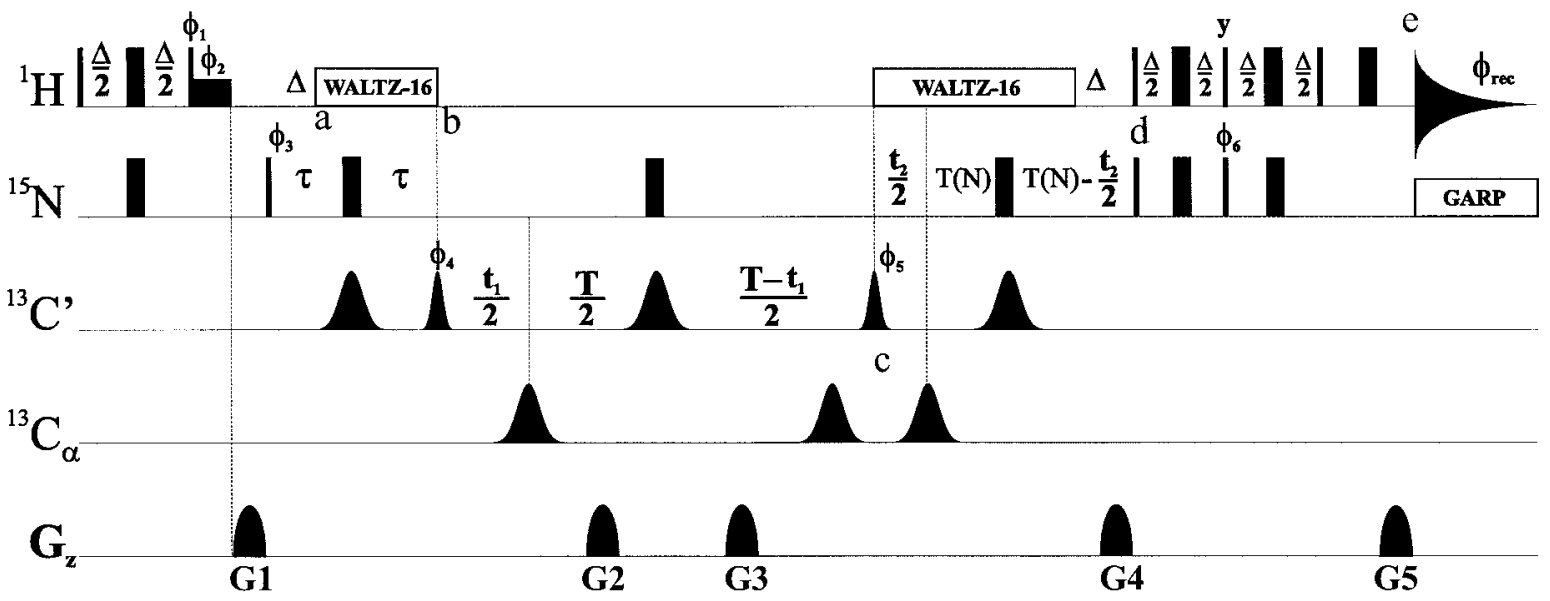

B

a Waltz-16

b

$D Q\left(N^{-} C^{-}\right): H_{z}^{N} \longrightarrow N^{+} H_{\alpha}^{N} e^{-\Gamma_{N, N H^{N}} \Delta-i \pi J_{N H^{N}} \Delta} \stackrel{(1-s)}{\longrightarrow} N^{-} H_{\alpha}^{N} e^{-\Gamma_{N, N H^{N}} \Delta-i \pi J_{N H^{*}} \Delta} \longrightarrow$
$D Q\left(N^{-} C^{-}\right): H_{z}^{N} \longrightarrow N^{+} H_{\beta}^{N} e^{+\Gamma_{N, N H^{N}} \Delta+i \pi J_{N H^{N}}} \stackrel{(1-s)}{\longrightarrow} N^{-} H_{\beta}^{N} e^{+\Gamma_{N, N H^{N}} \Delta+i \pi J_{N H^{N}} \Delta} \longrightarrow$

c Waltz-16

$$
\begin{aligned}
& N^{+} H_{\alpha}^{N} e^{-\Gamma_{N, N H^{N}} \Delta-i \pi J_{N H^{N}} \Delta} e^{-\Gamma_{D Q} T+i\left(\Omega_{N}+\Omega_{C}\right) t_{1}+i \pi\left(J_{N H^{N}}+J_{C^{\prime} H^{N}}\right) t_{1}} \\
& N^{+} H_{\beta}^{N} e^{+\Gamma_{N, N H^{N}} \Delta+i \pi J_{N H^{N}} \Delta} e^{+\Gamma_{D Q} T+i\left(\Omega_{N}+\Omega_{C}\right) t_{1}-i \pi\left(J_{N H^{N}}+J_{C^{\prime} H^{N}}\right) t_{1}} \underset{(1-s)}{\mathrm{d}} \\
& N H_{\alpha}^{N} e^{-2 \Gamma_{N, N N^{N}} \Delta} e^{-\Gamma_{D Q} T+i\left(\Omega_{N}+\Omega_{C}\right) t_{1}+i \pi\left(J_{N H^{N}}+J_{C H^{N}}\right) t_{1}} \longrightarrow H \\
& N^{+} H_{\beta}^{N} e^{+2 \Gamma_{N, N H^{N}} \Delta} e^{+\Gamma_{D Q} T+i\left(\Omega_{N}+\Omega_{C}\right) t_{1}-i \pi\left(J_{N H^{N}}+J_{C H^{N}}\right) t_{1}} \longrightarrow H
\end{aligned}
$$

FIG. 1. A: Pulse sequence for the measurement of the $\Gamma_{\mathrm{N}, \mathrm{NH} N}^{\mathrm{CSA} / \mathrm{DD}}+\Gamma_{\mathrm{C}^{\prime}, \mathrm{C}^{\prime} H^{N}}^{\mathrm{CSA}}$ and $\Gamma_{\mathrm{C}^{\prime}, \mathrm{NH}}^{\mathrm{CSA} / \mathrm{DD}}+\Gamma_{\mathrm{N}, \mathrm{C}^{\prime} \mathrm{H}^{N}}^{\mathrm{CSA} / \mathrm{DD}}$ rates $(6)$. The sequence is an HNCO correlation with the following parameters values: $\tau=13.5 \mathrm{~ms} ; \Delta=5.4 \mathrm{~ms} ; \phi_{1}=y ; \phi_{2}=-x ; \phi_{3}=x,-x ; \phi_{4}=2 x, 2(-x) ; \phi_{5}=4 x, 4(-x) ; \phi 6=y ; \phi_{\mathrm{rec}}=(x,-x$, $-x, x,-x, x, x,-x) ; \mathrm{G} 2=\mathrm{G} 3 ; \mathrm{G} 4=\left(\gamma_{\mathrm{H}} / \gamma_{\mathrm{N}}\right) \mathrm{G} 5$. All phases not explicitly given are equal to $x$. Quadrature detection is achieved in $t_{1}$ by States-TPPI on $\phi_{5}$, in $t_{2}$ by adding and subtracting two FIDs acquired with $\phi_{6}=y, \mathrm{G} 4=A$, and $\phi_{6}=-y, \mathrm{G} 4=-A$, respectively. ${ }^{13} \mathrm{C}$ spins are irradiated with selective pulses $\left(\mathrm{G} 4\left(90^{\circ}\right)=512 \mu \mathrm{s} ; \mathrm{Q} 3\left(180^{\circ}\right)=548.6 \mu \mathrm{s}\right)$. The power for proton decoupling is $4.1 \mathrm{kHz}$ and for nitrogen decoupling in acquisition $1.0 \mathrm{kHz}$. The proton carrier is set on water. The pulse with phase $\phi_{2}$ is a 2 -ms selective $\pi / 2$ pulse on water. Five experiments with different constant time values have been acquired ( $T=52,62,82,102$, and $122 \mathrm{~ms}$ ). B: Evolution of the DQ coherence $\mathrm{N}^{-} \mathrm{C}^{-}$for both the $\alpha$ and the $\beta$ state of the spin $\mathrm{H}_{\mathrm{N}}$ during the pulse sequence of A.

These represent a multiplet in antiphase with respect to the $J_{\mathrm{C}^{\prime} \mathrm{C}_{\alpha}}$ coupling in $t_{1}$, which causes the $1 / 2 \ln \left(\mathrm{In}_{0 \beta} / \mathrm{In}_{0 \alpha}\right)$ coefficient in Eq. [3] to be other than zero. This coefficient

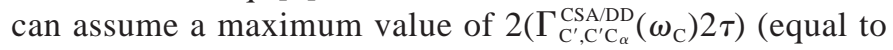
0.025 for a molecule with $\tau_{\mathrm{c}}=4.1 \mathrm{~ns}$ at $600 \mathrm{MHz}$ ). If a
$\Gamma_{\mathrm{C}^{\prime}, \mathrm{C}^{\prime} \mathrm{C}_{\alpha}}^{\mathrm{CSA} / \mathrm{D}}$ rate of $1.3 \mathrm{~Hz}$ was measured in a single experiment with a constant time of $100 \mathrm{~ms}$, the inaccuracy on the extracted value would be approximately $10 \%$. This error might be eliminated by a $90^{\circ} \mathrm{C}_{\alpha}$ pulse applied before the constant time $T$. 
A

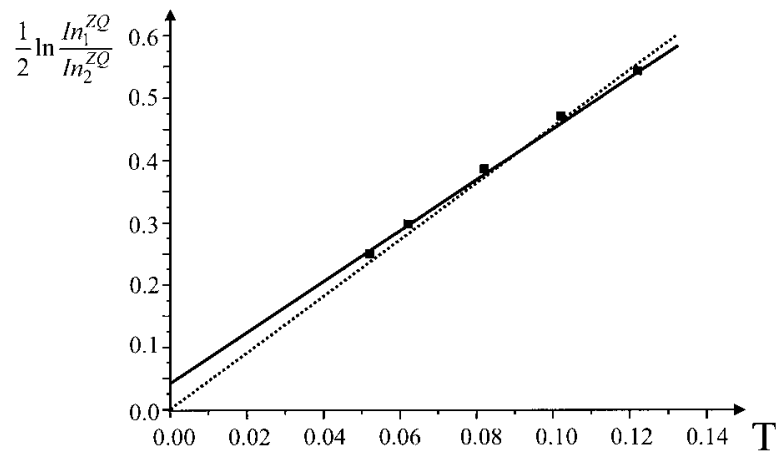

B

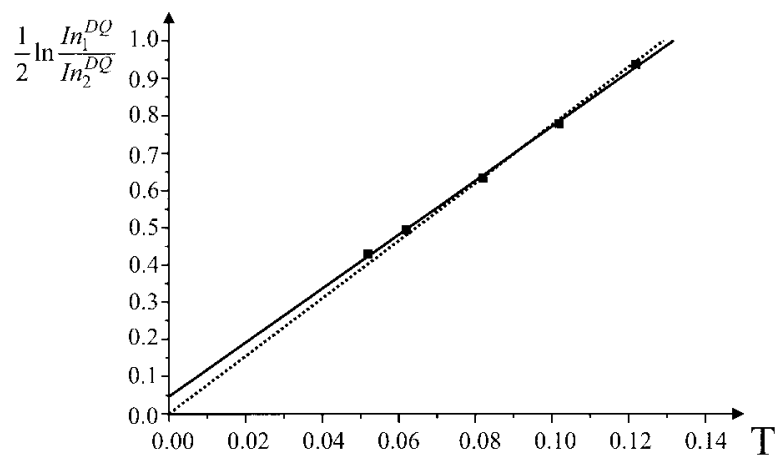

FIG. 2. Linear fitting of the data measured at different constant time values $T(52,62,82,102$, and $122 \mathrm{~ms})$ with the sequence of Fig. 1 according to Eq. [3]. (A) Fitting of $1 / 2 \ln \operatorname{In}_{\beta}^{Z Q} / \operatorname{In}_{\alpha}^{Z Q}$ for the peptide plane Lys29-Thr30: the continuous line corresponds to a fit according to Eq. [3], the dotted line to a linear fit with $1 / 2 \ln \left(\operatorname{In}_{0 \beta}^{\mathrm{ZQ}} / \mathrm{In}_{0 \alpha}^{\mathrm{ZQ}}\right)=0$; (B) Fitting of $1 / 2 \ln \left(\operatorname{In}_{\beta}^{\mathrm{DQ}} / \mathrm{In}_{\alpha}^{\mathrm{DQ}}\right)$ for the peptide plane Gly10-Lys11: the continuous line corresponds to a fit according to Eq. [3], the dotted line to a linear fit with $1 / 2 \ln \left(\operatorname{In}_{0 \beta}^{\mathrm{DQ}} / \mathrm{In}_{0 \alpha}^{\mathrm{DQ}}\right)=0$.

\section{Discussion}

The systematic errors, arising from noncomplete suppression of cross-correlated relaxation before and after the constant time period $T$, are present in quantitative $\Gamma$ experiments as well.
Sources of systematic errors can be eliminated experimentally suppressing all cross-correlated relaxation effects in delays other than $T$ or eliminating nondesired terms by purging pulses in both quantitative $\Gamma$ and $J$-resolved $\Gamma$ methods. If this is not practicable, accurate measurement of cross-correlated relaxation rates, even of small size, can be obtained by recording several experiments with different $T$ values and applying Eq. [3] to extract the desired rate.

\section{COMPARISON BETWEEN $J$-RESOLVED $\Gamma$ EXPERIMENTS AND QUANTITATIVE $\Gamma$ EXPERIMENTS}

Signal-to-noise ratio is one of the major factors affecting the accuracy of the measurement of NMR parameters. In the Appendix we present a detailed analysis of the statistical error for $\Gamma$ measured in both $J$-resolved and quantitative $\Gamma$ experiments. Moreover, we show how to optimize the relative duration of the cross and reference experiments in the quantitative $\Gamma$ method to obtain the smallest statistical error.

The sensitivity of the two methods can be compared by evaluating the calibrated relative error of the cross-correlated relaxation rate $\Gamma$ and the time $T, \Delta^{0}(\Gamma T) I_{\mathrm{r}}^{0} / \Gamma T \sigma$, from a $J$-resolved $\Gamma$ and a quantitative $\Gamma$ experiment of equal duration $t . I_{\mathrm{r}}^{0}$ is the signal intensity of a reference experiment of duration $t$ in the quantitative $\Gamma$ approach, or the sum of the intensities of the two multiplet lines in a $J$-resolved $\Gamma$ experiment of equal duration, and $\sigma$ is the noise associated with a spectrum acquired in the time $t$. The relative error $\Delta^{0}(\Gamma T) / \Gamma T$ depends on the measurement time $t$. On the other hand, the calibrated relative error $\Delta^{0}(\Gamma T) I_{\mathrm{r}}^{0} / \Gamma T \sigma$ is independent of the measurement time and represents the relative error $\Delta^{0}(\Gamma T) / \Gamma T$ calibrated with respect to the relative error $\sigma / I_{\mathrm{r}}^{0}$ of the signal $I_{\mathrm{r}}^{0}$ of an experiment of duration $t$. In Fig. 4 the two calibrated relative errors are plotted versus $\Gamma T$ for $J$-resolved and quantitative $\Gamma$ exper-

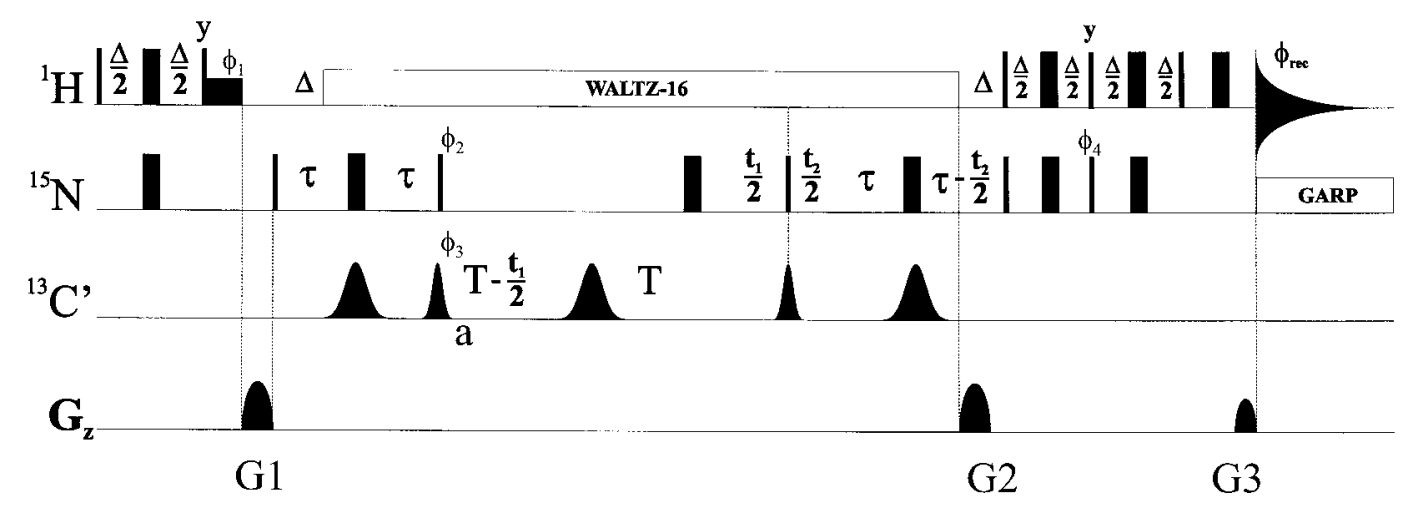

FIG. 3. Pulse sequence for the measurement of $\Gamma_{C^{\prime}, C^{\prime} C_{\alpha}}^{\mathrm{CS}}$ rate (7). The sequence is an HNCO correlation with the following parameters values: $\tau=13.5$ ms; $\Delta=5.4 \mathrm{~ms} ; \phi_{1}=-x ; \phi_{2}=x,-x ; \phi_{3}=x, x,-x,-x ; \phi_{4}=y ; \phi_{\text {rec }}=x,-x,-x, x ; \mathrm{G} 2=\left(\gamma_{\mathrm{H}} / \gamma_{\mathrm{N}}\right) \mathrm{G} 3$. All phases not explicitly given are equal to $x$. Quadrature detection is achieved in $t_{1}$ by States-TPPI on $\phi_{2}$, in $t_{2}$ by adding and subtracting two FIDs acquired with $\phi_{4}=y$, G2 $=A$, and $\phi_{4}=-y$, G2 $=$ $-A$, respectively. ${ }^{13} \mathrm{C}$ spins are irradiated with selective pulses $\left(\mathrm{G} 4\left(90^{\circ}\right)=512 \mu \mathrm{s} ; \mathrm{Q} 3\left(180^{\circ}\right)=548.6 \mu \mathrm{s}\right)$. The power for proton decoupling is $4.1 \mathrm{kHz}$ and for nitrogen decoupling in acquisition $1.0 \mathrm{kHz}$. The proton carrier is set on water. The pulse with phase $\phi_{1}$ is a 2 -ms selective $\pi / 2$ pulse on water. 


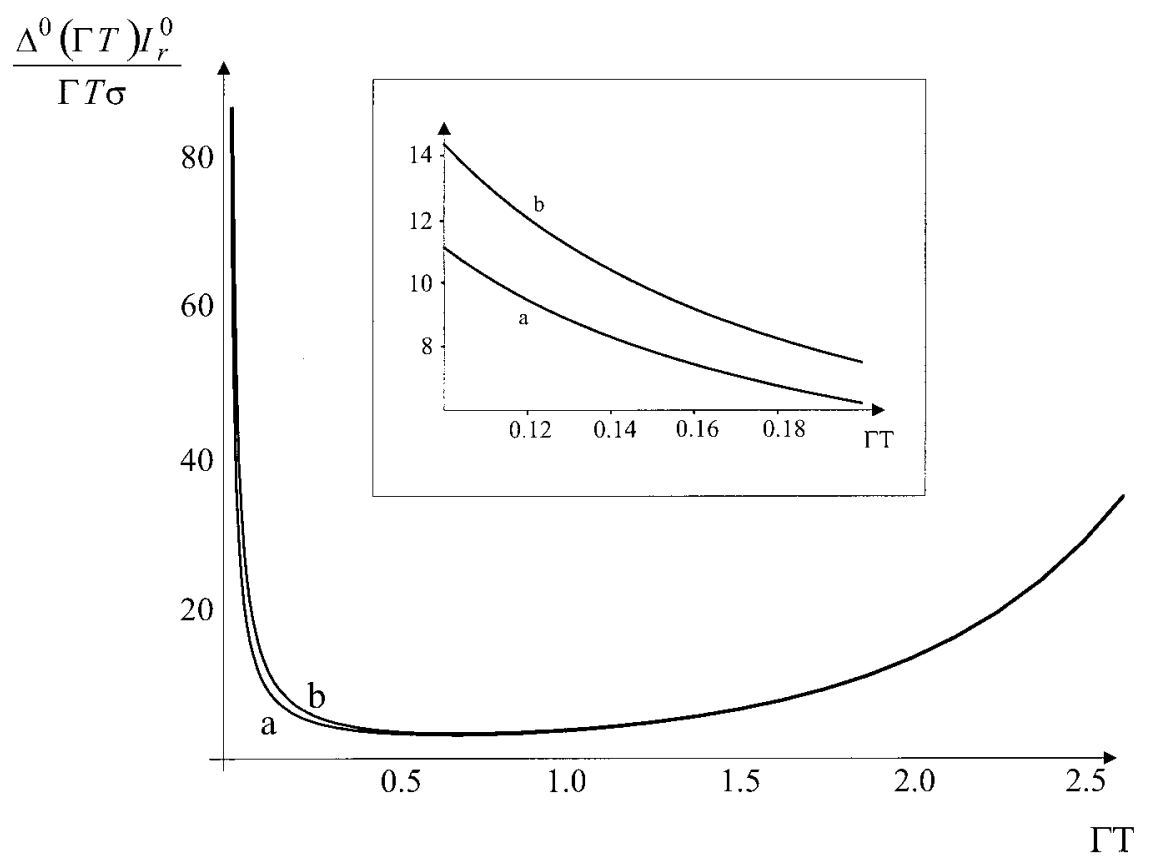

FIG. 4. Function $\Delta^{0}(\Gamma T)^{\text {quantit }} I_{\mathrm{r}}^{0} / \sigma \Gamma T$ (line a) and function $\Delta^{0}(\Gamma T)^{J \text {-resolved }} I_{\mathrm{r}}^{0} / \sigma \Gamma T$ for a $J$-resolved multiplet consisting of two lines (line b) plotted versus $\Gamma T$ for $\Gamma T=0-2.5$. The error derived from quantitative $\Gamma$ experiments is smaller than the one derived from a $J$-resolved $\Gamma$ experiment for low $\Gamma T$. For $\Gamma T>$ 0.5 the two functions become equal. In the inset, an expansion of the plot for $\Gamma T=0.1-0.2$ shows that for $\Gamma T \ll 1$ the difference between the two errors is equal to a factor of $\sqrt{2}$.

iments. $\Delta^{0}(\Gamma T)^{\text {quantit }} I_{\mathrm{r}}^{0} / \Gamma T \sigma$ is the calibrated relative error of $\Gamma T$ in a quantitative $\Gamma$ approach where the sum of the duration of the cross and the reference experiments is $t$; $\Delta^{0}(\Gamma T)^{J-\text { resolved }} I_{\mathrm{r}}^{0} / \Gamma T \sigma$ is the calibrated relative error of $\Gamma T$ for a $J$-resolved $\Gamma$ experiment of duration $t$. For $\Gamma T \ll 1$, the error $\Delta^{0}(\Gamma T) I_{\mathrm{r}}^{0} / \Gamma T \sigma$ (Eq. [A15]) is smaller in the quantitative $\Gamma$ method than in the $J$-resolved $\Gamma$ method (Eq. [A16]). The ratio between the two relative errors is expected to be equal to $\sqrt{2}$ if the multiplet in the $J$-resolved $\Gamma$ experiment consists of two lines, as can be seen in Fig. 4 (the ratio becomes 2 for a $J$-resolved $\Gamma$ experiment where the multiplet consists of four lines). Thus, for $\Gamma T<0.5$ the relative error of $\Gamma T$ in the quantitative $\Gamma$ approach is at least a factor of $\sqrt{2}$ smaller than that in the $J$-resolved $\Gamma$ approach. However, for $\Gamma T>0.5$, the two relative errors become equal and the two methods (for $J$-resolved multiplets consisting of two lines) yield equivalent results (Fig. 4). The condition $\Gamma T>0.5$ can be met when the cross-correlated relaxation rate $\Gamma$ is of the same order of magnitude as the autocorrelated transversal relaxation rate.

In general, whenever a cross-correlated relaxation rate can be measured with both strategies, the quantitative $\Gamma$ experiment is to be preferred, because of the higher signal to noise, for $\Gamma T<0.5$. However, $J$-resolved $\Gamma$ experiments with four multiplet lines usually contain information about three different cross-correlated relaxation rates. Extracting several rates from the same experiment is a major advantage, as it ensures exactly equal experimental conditions for all measured parameters.

\section{CHOOSING APPROPRIATE CONSTANT TIME DELAYS FOR THE EVOLUTION OF CROSS-CORRELATED RELAXATION RATES}

The optimal constant time $T$ in the quantitative $\Gamma$ and in $J$-resolved $\Gamma$ experiments is the one which gives the smallest relative error for the measured parameter $\Gamma T$. To calculate the most appropriate value, relaxation of the transverse magnetization present during the constant time $T$ must be taken into account. In practice, in the analytical expression for the calibrated relative error, the signal intensity $I_{\mathrm{r}}^{0}$ of a reference experiment of duration $t$ (see Appendix) must be substituted by $I_{\mathrm{r}}^{0} e^{-R_{2} T}$, where $R_{2}$ is the autocorrelated transversal relaxation rate of the transversal operator present during the time $T$. Assuming $\Gamma T \ll 1$, the relative errors are given by

$$
\begin{gathered}
\frac{\Delta^{0}(\Gamma T) I_{\mathrm{r}}^{0}}{\Gamma T \sigma}=\frac{\sqrt{2} e^{R_{2} T}}{\Gamma T} \text { for the } J \text {-resolved } \Gamma \text { experiment } \\
\frac{\Delta^{0}(\Gamma T) I_{\mathrm{r}}^{0}}{\Gamma T \sigma}=\frac{e^{R_{2} T}}{\Gamma T} \quad \text { for the quantitative } \Gamma \text { experiment. }
\end{gathered}
$$


The minima of the two functions in Eq. [7] are found at

$$
\begin{aligned}
T= & \frac{1}{R_{2}} \text { for both the } J \text {-resolved } \Gamma \text { experiment and the } \\
& \text { quantitative } \Gamma \text { experiment. }
\end{aligned}
$$

For $\Gamma T \geq 1$, Eqs. [A15] and [A16] (Appendix) must be used. In this case the optimal value for $T$ cannot be calculated analytically, but can be extracted from a graphical presentation of Eq. [9]:

$$
\begin{aligned}
& T\left(R_{2}+\Gamma\left(\tanh 2 \Gamma T\left(1+\frac{\cosh 2 \Gamma T}{1+\cosh 2 \Gamma T}\right)\right)\right)=1 \\
& \quad \text { for the } J \text {-resolved } \Gamma \text { experiment } \\
& T\left(R_{2}+\Gamma\left(2 \tanh \Gamma T+\frac{1}{\cosh ^{2} \Gamma T(1+\tanh \Gamma T)}\right)\right)=1
\end{aligned}
$$

for the quantitative $\Gamma$ experiment.

For $R_{2}=20 \mathrm{~Hz}$ and $\Gamma=0.1 \mathrm{~Hz}$, for example, the two functions of Eq. [9] nearly coincide and the best constant time $T$ is found at $50 \mathrm{~ms}$, as expected from Eq. [8] for $\Gamma T \ll 1$. For $R_{2}=30 \mathrm{~Hz}$ and $\Gamma=20 \mathrm{~Hz}$, the two functions of Eq. [9] are also very close in the interesting area, but the optimal value of $T$ (20 ms) significantly deviates from what would be calculated from Eq. [8].

\section{CONCLUSIONS}

We have analyzed two $J$-resolved $\Gamma$ experiments and found that evolution of cross-correlated relaxation effects before and after the constant time period $T$ is a source of systematic errors. This statement is general and applies to quantitative $\Gamma$ experiments as well. If the systematic errors are not eliminated by appropriate purging, they can be removed by recording several experiments with different constant time values and by fitting the data to a linear function of the form $f(T)=A+B T$ with $A \neq 0$. In addition, we have derived analytical expressions for the statistical errors in both $J$-resolved and quantitative $\Gamma$ experiments. These calculations assist in the choice of the most appropriate method for measuring a certain rate and in estimating the accuracy of the results. Moreover, the optimal value for the constant time $T$ can be calculated for both methods and depends on the relaxation properties of the system under investigation.

\section{APPE NDIX}

Let $(a+1) t$ be the time that can be invested in the measurement of the cross-correlated relaxation rate $\Gamma$ with the quantitative $\Gamma$ method. We define the experimental time $t$ as the time necessary to reach the noise level $\sigma$ in the spectrum.
$I_{\mathrm{r}}^{0}$ and $I_{\mathrm{c}}^{0}$ are the intensities of the signal of a reference and cross experiment, each of duration $t$.

Generally, the error $\Delta f\left(x_{1}, x_{2}, \ldots\right)$ on a function $f\left(x_{1}\right.$, $\left.x_{2}, \ldots\right)$ is given by

$$
\begin{aligned}
& \Delta f\left(x_{1}, x_{2}, \ldots\right) \\
& =\sqrt{\begin{array}{l}
\left(\frac{\partial f\left(x_{1}, x_{2}, \ldots\right)}{\partial x_{1}}\right)^{2} \Delta^{2} x_{1} \\
+\left(\frac{\partial f^{\prime}\left(x_{1}, x_{2}, \ldots\right)}{\partial x_{2}}\right)^{2} \Delta^{2} x_{2}^{2}+\ldots
\end{array}} .
\end{aligned}
$$

The error $\Delta(\Gamma T)^{\text {quantit }}$ of $(\Gamma T)^{\text {quantit }}$, which is the value of $\Gamma T$ obtained in the quantitative $\Gamma$ approach and is a function of $I_{\mathrm{r}}^{0}$ and $I_{\mathrm{c}}^{0}$, is given, according to the error propagation formula A1:

$$
\Delta(\Gamma T)^{\text {quantit }}=\sqrt{\left(\frac{\partial(\Gamma T)}{\partial I_{\mathrm{r}}^{0}}\right)^{2} \Delta^{2} I_{\mathrm{r}}^{0}+\left(\frac{\partial(\Gamma T)}{\partial I_{\mathrm{c}}^{0}}\right)^{2} \Delta^{2} I_{\mathrm{c}}^{0}}
$$

If the duration of the cross experiment is at and that of the reference experiment $t$, for a total measurement time of $(a+$ 1) $t$, and remembering that $\Gamma T \approx \tanh \Gamma T=I_{\mathrm{c}} / a I_{\mathrm{r}}=I_{\mathrm{c}}^{0} / I_{\mathrm{r}}^{0}$, we find

$$
\Delta(\Gamma T)^{\text {quantit }}=\sigma \Gamma T \sqrt{\frac{1}{a I_{\mathrm{c}}^{0^{2}}}+\frac{1}{I_{\mathrm{r}}^{0^{2}}}},
$$

where it has been assumed that the error of the signal in the cross experiment of duration at is $\sqrt{a} \sigma$, that the intensity of the signal is $I_{\mathrm{c}}=a I_{\mathrm{c}}^{0}$ and $I_{\mathrm{r}}=I_{\mathrm{r}}^{0}$, and that $\tanh (\Gamma T) \approx \Gamma T$ (which is true for $\Gamma T \ll 1$ ). In order to find the best value of $a$, namely the best ratio between the duration of the cross and reference experiments, we define the error $\Delta^{0}(\Gamma T)$ as the error of the variable $\Gamma T$ measured with the quantitative $\Gamma$ method, if the total duration of both cross and reference experiments is equal to $t$. This corresponds to normalizing the calculation to a certain experimental time $t$ during which an amount of noise equal to $\sigma$ is introduced in the spectrum. This normalized error is given by Eq. [A3] multiplied by $\sqrt{a+1}$, because the intensities of the signals $I_{\mathrm{r}}^{0}$ and $I_{\mathrm{c}}$ are scaled down by a factor $(a+1)$, while the noise is scaled down by a factor of $\sqrt{a+1}$ only:

$$
\Delta^{0}(\Gamma T)^{\text {quantit }}=\sigma \Gamma T \sqrt{\frac{1}{a I_{\mathrm{c}}^{0^{2}}}+\frac{1}{I_{\mathrm{r}}^{0^{2}}}} \sqrt{a+1} .
$$

This expression can be derived with respect to $a$ to find the minimum. The value one obtains is

$$
a=\frac{I_{\mathrm{r}}^{0}}{I_{\mathrm{c}}^{0}} \approx \frac{1}{\Gamma T},
$$


where it has been assumed that $\tanh (\Gamma T) \approx \Gamma T$. After substitution of Eq. [A5] into Eq. [A3], one finds

$$
\Delta(\Gamma T)^{\text {quantit }}=\frac{\sigma \Gamma T}{I_{\mathrm{r}}^{0}} \sqrt{\frac{1}{\Gamma T}+1}
$$

In a corresponding $J$-resolved $\Gamma$ experiment of duration $(a+$ 1) $t$, whose multiplets consist of two lines with intensities $I_{1}$ and $I_{2}$, the error on $\Gamma T\left(\Gamma T=1 / 2 \ln \left(I_{1} / I_{2}\right)\right)$, is

$$
\begin{aligned}
\Delta(\Gamma T)^{J \text {-resolved }} & =\sqrt{\left(\frac{\partial(\Gamma T)}{\partial I_{1}}\right)^{2} \Delta^{2} I_{1}+\left(\frac{\partial(\Gamma T)}{\partial I_{2}}\right)^{2} \Delta^{2} I_{2}} \\
& =\frac{\sigma \sqrt{(1+a)}}{2} \sqrt{\frac{1}{I_{1}^{2}}+\frac{1}{I_{2}^{2}}}
\end{aligned}
$$

where the noise of the experiment is $\sigma \sqrt{(1+a)}$. If $\Gamma T$ is very small,

$$
I_{1} \approx I_{2} \approx \frac{(a+1)}{2} I_{\mathrm{r}}^{0}
$$

which gives from Eq. [A7], substituting $a=1 / \Gamma T$,

$$
\Delta(\Gamma T)^{J \text {-resolved }}=\frac{\sqrt{2 \Gamma T} \sigma}{I_{\mathrm{r}}^{0} \sqrt{1+\Gamma T}} .
$$

Under the assumption that $\Gamma T \ll 1$, Eq. [A6] becomes

$$
\Delta(\Gamma T)^{\text {quantit }}=\frac{\sigma \sqrt{\Gamma T}}{I_{\mathrm{r}}^{0}}
$$

and Eq. [A9]

$$
\Delta(\Gamma T)^{J \text {-resolved }}=\frac{\sigma \sqrt{2 \Gamma T}}{I_{\mathrm{r}}^{0}}=\sqrt{2} \Delta(\Gamma T)^{\text {quantit }},
$$

from which the expected factor of $\sqrt{2}$ between the error of $\Gamma T$ in the quantitative $\Gamma$ method (Eq. [A10]) and that in the $J$-resolved $\Gamma$ method (Eq. [A11]) is found. If the multiplets of the $J$-resolved $\Gamma$ experiment consist of four lines instead of two, Eq. [A11] becomes

$$
\Delta(\Gamma T)^{J \text {-resolved }}=\frac{2 \sigma \sqrt{\Gamma T}}{I_{\mathrm{r}}^{0}}=2 \Delta(\Gamma T)^{\text {quantit. }} .
$$

If the assumption $\Gamma T \ll 1$ is not true, Eq. [A3] becomes, after substituting $a=I_{\mathrm{r}}^{0} / I_{\mathrm{c}}^{0}$,

$$
\begin{aligned}
\Delta(\Gamma T)^{\text {quantit }} & =\frac{\sigma \cosh ^{2}(\Gamma T) \tanh (\Gamma T)}{I_{\mathrm{r}}^{0}} \sqrt{1+\frac{I_{\mathrm{r}}^{0}}{I_{\mathrm{c}}^{0}}} \\
& =\frac{\sigma \cosh ^{2}(\Gamma T) \tanh (\Gamma T)}{I_{\mathrm{r}}^{0}} \sqrt{1+\frac{1}{\tanh (\Gamma T)}}
\end{aligned}
$$

and Eq. [A5], after substituting $I_{1,2}=\left(1 / 2 I_{\mathrm{r}}^{0}(a+1) e^{ \pm \Gamma T}\right) /$ $\cosh (\Gamma T)$,

$$
\Delta(\Gamma T)^{J \text {-resolved }}=\frac{\sigma \cosh (\Gamma T)}{I_{\mathrm{r}}^{0} \sqrt{1+\frac{1}{\tanh \Gamma T}}} \sqrt{e^{2 \Gamma T}+e^{-2 \Gamma T}} .
$$

The relative errors per time $t$ are given by

$$
\begin{aligned}
\frac{\Delta^{0}(\Gamma T)^{\text {quantit }}}{\Gamma T} & =\frac{\sigma \cosh ^{2}(\Gamma T) \tanh (\Gamma T)}{I_{\mathrm{r}}^{0} \Gamma T}\left(\frac{I_{\mathrm{r}}^{0}}{I_{\mathrm{c}}^{0}}+1\right) \\
& =\frac{\sigma \cosh ^{2}(\Gamma T)}{I_{\mathrm{r}}^{0} \Gamma T}(1+\tanh (\Gamma T))
\end{aligned}
$$

$$
\frac{\Delta^{0}(\Gamma T)^{J \text {-resolved }}}{\Gamma T}=\frac{\sigma \cosh (\Gamma T)}{I_{\mathrm{r}}^{0} \Gamma T} \sqrt{e^{2 \Gamma T}+e^{-2 \Gamma T}} .
$$

The values of $\left(\Delta^{0}(\Gamma T)^{\text {quantit }} / \Gamma T\right)\left(I_{\mathrm{r}}^{0} / \sigma\right)$ and $\left(\Delta^{0}(\Gamma T)^{J \text {-resolved/ }}\right.$ $\Gamma T)\left(I_{\mathrm{r}}^{0} / \sigma\right)$ are reported versus $\Gamma T$ in Fig. 4 , where it is evident that the two calibrated relative errors become equal for $\Gamma T>$ 0.5 .

\section{REFERENCES}

1. B. Reif, M. Hennig, and C. Griesinger, Direct measurement of angles between bond vectors in high-resolution NMR, Science 276, 1230-1233 (1997).

2. B. Reif, H. Steinhagen, B. J unker, M. Reggelin, and C. Griesinger, Determination of the orientation of a distant bond vector in a molecular reference frame by cross-correlated relaxation of nuclear spins, Angew. Chem. Int. Ed. Eng. 37, 1903-1906 (1998).

3. C. Griesinger, M. Hennig, J . P. Marino, B. Reif, and H. Schwalbe, in "Modern Methods in Protein NMR," Vol. 16 (L. J . Berliner and N. R. Krishna, Eds.), Plenum, London, 1999.

4. D. Yang and L. E. Kay, Determination of the protein backbone dihedral angle from a combination of NMR-derived cross-correlation spin relaxation rates, J. Am. Chem. Soc. 120, 9880-9887 (1998).

5. D. Yang, R. Konrat, and L. E. Kay, A multidimensional NMR experiment for measurement of the protein dihedral angle based on cross-correlated relaxation between ${ }^{1} \mathrm{H}-{ }^{13} \mathrm{C}$ dipolar and ${ }^{13} \mathrm{C}^{\prime}$ (carbonyl) chemical shift anisotropy mechanisms, J. Am. Chem. Soc. 119, 11938-11940 (1997).

6. B. Brutscher, N. R. Skrynnikov, T. Bremi, R. Brüschweiler, and R. R. Ernst, Quantitative investigation of dipole-CSA cross-correlated relaxation by ZQ/DQ spectroscopy, J. Magn. Reson. 130, 346-351 (1998).

7. M. W. F. Fischer, L. Zeng, Y. Pang, W. Hu, A. Majumdar, and 
E. R. P. Zuiderweg, Experimental characterization of models for backbone picosecond dynamics in proteins. Quantification of NMR auto- and cross-correlation relaxation mechanisms involving different nuclei of the peptide plane, J. Am. Chem. Soc. 119, 1262912642 (1997).

8. N. Tjandra, A. Szabo, and A. Bax, Protein backbone dynamics and ${ }^{15} \mathrm{~N}$ chemical shift anisotropy from quantitative measurement of relaxation interference effects, J. Am. Chem. Soc. 118, 6986-6991 (1996).

9. M. Tessari, H. Vis, R. Boelens, R. Kaptein, and G. W. Vuister, Quantitative measurement of relaxation interference effects between ${ }^{15} \mathrm{~N}$ CSA and ${ }^{1} \mathrm{H}-{ }^{15} \mathrm{~N}$ dipolar interaction: Correlation with secondary structure, J. Am. Chem. Soc. 119, 8985- 8990 (1997).

10. C. D. Kroenke, J. P. Loria, L. K. Lee, M. Rance, and A. G. Palmer, III, Longitudinal and transverse ${ }^{1} \mathrm{H}-{ }^{15} \mathrm{~N}$ dipolar $/{ }^{15} \mathrm{~N}$ chemical shift anisotropy interference: Unambiguous determination of rotational diffusion tensors and chemical exchange effects in biological macromolecules, J. Am. Chem. Soc. 120, 7905-7915 (1998).
11. I. C. Felli, C. Richter, C. Griesinger, and H. Schwalbe, Determination of RNA sugar pucker mode from cross-correlated relaxation in solution NMR spectroscopy, J. Am. Chem. Soc. 121, 1956-1957 (1999).

12. T. Carlomagno, I. C. Felli, M. Czech, R. Fischer, M. Sprinzl, and C. Griesinger, Transferred cross-correlated relaxation: Application to the determination of sugar pucker in an aminoacylated tRNAmimetic weakly bound to EF-Tu, J. Am. Chem. Soc. 121, 19451948 (1999).

13. P. Pelupessy, E. Chiarparin, R. Ghose, and G. Bodenhausen, Efficient determination of angles subtended by $\mathrm{C}_{\alpha}-\mathrm{H}_{\alpha}$ and $\mathrm{NH}^{\mathrm{N}}$ vectors in proteins via dipole- dipole cross-correlation, J. Biomol. NMR 13, 375-380 (1999).

14. E. Chiarparin, P. Pelupessy, R. Ghose, and G. Bodenhausen, Relaxation of two-spin coherence due to cross-correlated fluctuations of dipole-dipole couplings and anisotropic shifts in NMR of ${ }^{15} \mathrm{~N},{ }^{13} \mathrm{C}$-labeled biomolecules, J. Am. Chem. Soc. 121, $6876-6883$ (1999). 